\title{
Fireflies Ant Optimized Reliable Quality Awareness Energy Efficient Routing Protocol for Managing Quality of Medical Data in Wireless Body Networks
}

\author{
M.Santhalakshmi, P.Kavitha
}

\begin{abstract}
The wireless body area network is one of effective wearable devices that have been used in medical applications for collecting patient information to providing the treatment incorrect time for avoiding seriousness. The collected data's such as blood pressure, air flow, temperature, electromagnetic information is transmitted to the health care center via the wireless technology, which reduces the difficulties also helps to provide the immediate treatment. During the information transmission, the main issues are Quality of Service (QoS), low packet delivery, high energy consumption and end to end delay. So, in this paper introduces the Fireflies Ant Optimized, Reliable Quality Awareness, Energy Efficient Routing Protocol ((FAORQEER) for maintaining the quality of the recorded medical data. The network examines the optimal path by using the characteristics of fireflies and the network life time and energy of the network is managed by introducing an energy efficient method. The process then evaluates efficiency with test results about energy consumption, packet delivery ratio, end to end delay and QoS metric associated constraints.
\end{abstract}

Keywords:Wireless body area network, Medical applications, Quality of Service (QoS), Fireflies Ant optimized reliable quality awareness energy efficient routing protocol.

\section{INTRODUCTION}

The development of technologies effectively utilized in the medical field because patient information's are need to be collected successfully for giving the treatment with right time. Due to the importance of the patient information, Biomedical Wireless Sensor Network (BWSN) is utilized for collecting the information from human begins that is achieved with the help of wearable sensor device [1]. The placed sensor device continuously monitors the human begins electrical activities and related electromagnetic deviations which helps to capture several information's such as blood pressure, temperature, heart rate, EEG, $\mathrm{SpO} 2$, ECG, EMG and pulse data. These captured images are biomedical information [2] is broadcast to the nearby health care centers for examining the patient conditions in that situation. During the bio-sensor data transmission process, it has been examined in terms of emergency data, sensor information, location, the similar data is grouped and it has been divided based on the importance [3]

Revised Manuscript Received on November 08, 2019.

M.Santhalakshmi, Assistant Professor, Department of Computer Science, Sri Kailash Womens College,Thalaivasal-636112.

P.Kavitha

Head, Department of Computer Science, Pavendhar College of Arts \& Science, M.South, Attur-636102
Such as ordinary packet, middle range packet and emergency packets because it used to enhance the quality of transmitting data with effective manner. The divided data have been transmitted from source to destination by choosing an optimized route because; the router decides the data privacy, quality, authentication and other quality related factors. These quality factors are leads to create route failure, node failure, link failure and data consistency issue. So, different routing technologies [4] such as energy routing protocol, quality based routing protocol, AODV routing protocol are used to transmit the data from source to destination. There are several researches are analyze different routing protocols for transmitting data from source to destination place. Biomedical Wireless Sensor Networks (BWSN) [5] is put an essential part while observing the patient exercises from any area. Different exchanges have been done in the field of BWSN based patient observing procedure. Xiaochen Lai, et al.,[6] centering the information combination and system correspondence based framework for checking the patient exercises by using the ideas of the body sensor organize innovation. The creator gathers the signs from the different patients by putting the hub on the human body which examines the data with different bearings that used to lessens the different issues. In future, the creator makes the multi innovation based framework for keeping up the nature of the administrations while transmitting the data in the system with proficient way. Javed Iqbal Bangash et al.,[7] examining the different challenges introduce in the remote sensor body zone organize while transmitting the patient data in the remote system. Amid the information transmission, the directing is the most critical factor, on the grounds that steering conventions distinguish the ideal way between the sources to goal. At last the productivity of the different steering convention in the WBSN is examined as far as their quality and shortcoming parameter. Zahoor Khan et al.,[8] building up the vitality mindful peering steering convention for making the productive correspondence in the indoor clinic condition. The strategy build the steering table with the assistance of the companion disclosure process which deal with the vitality utilization, arrange life time, activity stack additionally enhances the body territory organize dependability. At that point the execution of the framework is actualized with the assistance of the Castalia recreation instrument which accomplishes the productive outcomes while transmitting the information in the system. Also the created framework diminishes the general lower vitality utilization when contrasted with alternate conventions Although these routing protocol ensures the successful route, the network still face 
several issues [9] such as power consumption, energy, resource utilization that will completely entire bio sensor data transmission process and packet delivery ratio. These discussed issues are overcome by introducing the Fireflies Ant optimized reliable quality awareness energy efficient routing protocol ((FAORQEER) which manages the network energy and power issues because optimal path by using the characteristics of fireflies. Further the network life time and energy of the network is managed by introducing energy efficient method. The energy efficient method examines the each node and related path is selected based on the reliability value. The defined reliability value is computed depending on the neighboring node information and relevant average weighted factor value. From the determined energy value, the network life time is examined that used to maintain the network lifetime with effective manner which used to transmit the bio-sensor data from source to destination. At last the efficiency of the Fireflies Ant optimized reliable quality awareness energy efficient routing protocol ((FAORQEER) is evaluated in terms if using simulation results and discussions.

\section{Fireflies Ant Optimized Reliable Quality Awareness Energy Efficient Routing Protocol}

In this section analyze the Fireflies Ant optimized reliable quality awareness energy efficient routing protocol (FAORQEER) based bio-sensor data transmission in biomedical body sensor network. The structure of the energy efficient routing process is shown in figure 1.
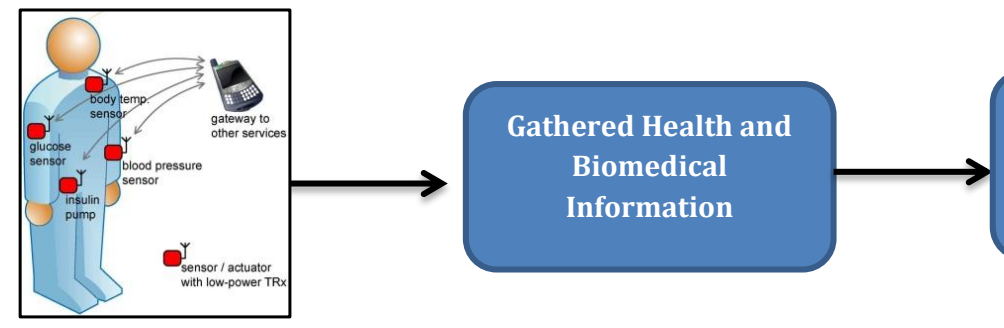

Divided the packet

into ordinary, middle

and emergency

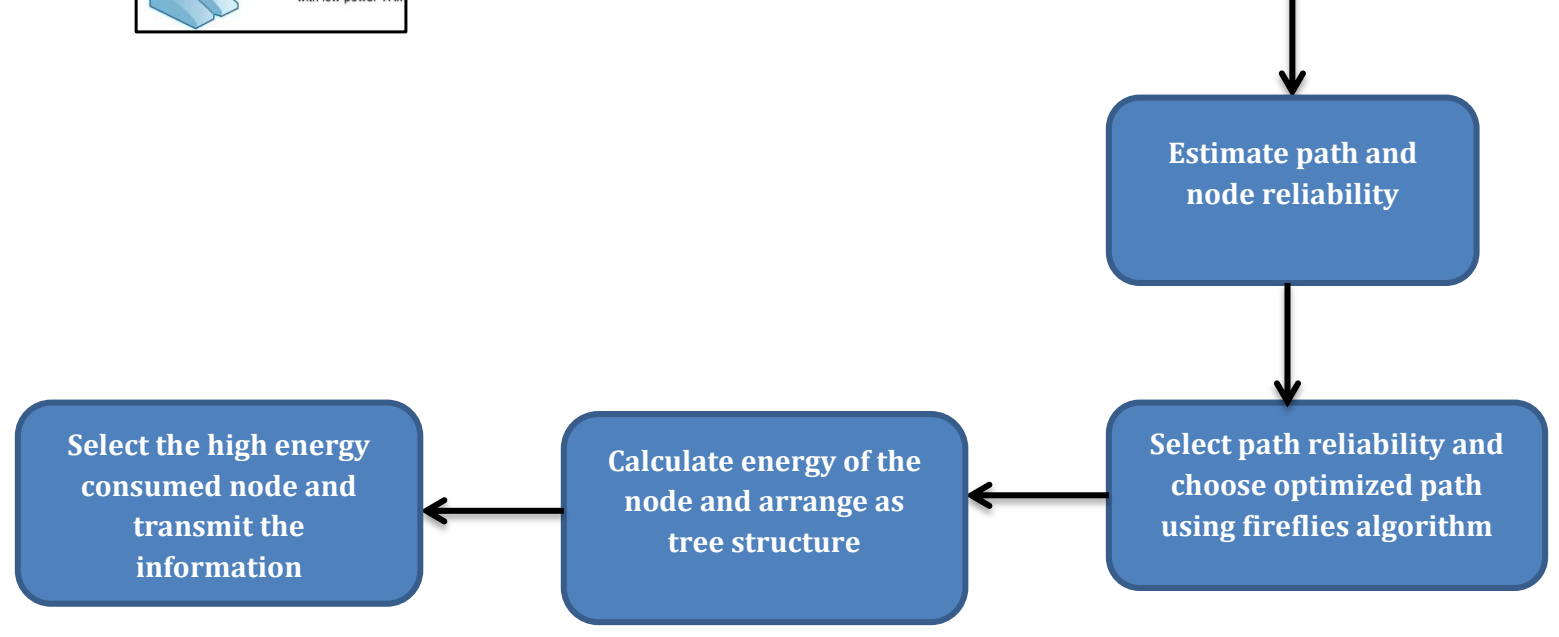

Figure 1: FAORQEER based Information Routing Process

First the wearable sensor device [10] is placed a human body that collects the patient information which is transmit to health care center for providing treatment to the patient. The collected data is transmitted to destination by selecting the optimized and reliable path because it used to avoid the intermediate attacks also maintain the reliability of the sensitive packets [11]. At the time of path reliability computation process, the neighboring node table has been maintained for managing the path reliability. Initially, the source node is examined along with reliability value and it has been compared with the neighboring node reliability value that is obtained from the neighboring node information. The obtained two reliability value is compared one other, if it is lower than the computed reliability value[12], and the greater reliability path is selected for transmitting data from source to destination. $\boldsymbol{R}_{\text {path }(i, D i s)=} \boldsymbol{R}_{\text {link }(i, j)} * \boldsymbol{R}_{\text {path }(j, D i s t)}$

In the eqn (1),

i is source node, Dis is destination node

The node reliability value is determined as follows, based on the path reliability value.
$R_{\operatorname{link}(i, j)}=(1-\propto) R_{\operatorname{link}(i, j)}+\propto X_{i}$

In the eqn (2),

\section{$\propto$ is represented as the average weighting factor of the node}

Depending on the route reliability value, the node reliability value is determined as follows.

$X_{i}=\frac{N_{\text {acks }}}{N_{\text {Trans }}}$

In the above eqn (3),

$$
\boldsymbol{R}_{\text {path(i,Dis) }} \text { is the reliability path between }
$$
node $i$ to destination

$R_{\text {link }(i, j)}$ is the link between node $i$ and $j$.

The average weighting factor is 0.4 ,

$N_{\text {acks }}$ is represented as the number of acknowledgement received for packets

$N_{\text {Trans }}$ is denoted as the number of packet transmitted.

The selected path is further optimized by applying the fireflies' ant approach [13] because it used to detect the optimized and reliable path from source to destination with effective manner. From 
the selected path, its probability value is computed as follows,

$$
P_{i}^{k}(t)=\left\{\frac{\left|t_{i}(t)\right|^{\alpha}\left|n_{j}\right|^{\beta}}{\sum_{\mu}\left|t_{i}(t)\right|^{\alpha}\left|n_{j}\right|^{\beta}} \quad \text { if } i \boldsymbol{j}^{k}\right.
$$

where $\boldsymbol{j}^{\boldsymbol{k}}$ is the set of selected path information, $\mathrm{t}$ and $\mathrm{n}$ are the reliability value of node and link, and $\alpha, \beta$ is the heuristic information. Based on the probability value, distance between two selected path is computed by Minkowski distance measure [14] that helps to determine the attractiveness or quality of the path that is estimated as follows.

$$
\text { attractiveness }=\left(\sum_{i=1}^{n}\left|x_{i}-y_{i}\right|^{p}\right)^{1 / p}
$$

Where $\boldsymbol{n}$ represents the number of selected path set and $\boldsymbol{p}$ is the Minkowski distance inequality values. After computing the quality of the path, the direction change of path is examined as follows.

$F_{i j}^{d}=G(t) \frac{M_{i}(t) * M_{j}(t)}{\left(D_{i j}(t)\right)^{n}+\varepsilon}$

In the above Eq. (6), $\boldsymbol{F}_{\boldsymbol{i} j}^{\boldsymbol{d}}$ represents the magnitude of the path on $\mathrm{i}$ and $\mathrm{j}$ in the $\mathrm{d}^{\text {th }}$ dimension.

$\mathrm{G}(\mathrm{t})$ is the gravitational force in a given time $\mathrm{t} \boldsymbol{M}_{\boldsymbol{i}}(\boldsymbol{t})$ and $\boldsymbol{M}_{\boldsymbol{j}}(\boldsymbol{t})$ are the two different path's mass values. $\boldsymbol{D}_{\boldsymbol{i j}}(\boldsymbol{t})$ is the distance from the two roads. The optimized path from the computed path information is chosen based on the following fitness value,

$$
F_{i}^{d}(t)=\sum_{j \epsilon k-b e s t} j \neq i \operatorname{rand}_{j} F_{i j}^{d}(t)
$$

In the above Eq. (7), $\boldsymbol{r a n d}_{\boldsymbol{j}}$ is a random number with a value between 0 and 1 . The route and link are selected based on the aforementioned discussions, where sensible data are effectively transmitted from source to destination. Together with this chosen path, node energy use will be determined to prevent a connection failure, node failure, network failure, etc. Next, the whole node in the network and the distance from origin to destination must be controlled. The energy factor for choosing the next hop value from the neighboring table is considered from the measured distance calculation.In addition to this link cost value is computed as follows,

$\boldsymbol{C}_{u v}=\min \left\{R E_{u}-E_{t x}, R E_{v}-E_{r x}\right\}$

In the eqn (8),

\section{$E_{t x}$ is represented as the node transmission cost} $E_{r x}$ is the reception cost for node

$R E_{u}$ and $R E_{v}$ is residual energy of the node.

Based on the link cost, residual energy value, the selected next hop nodes are ordered in the tree structure that helps to transmit the bio-sensor data without any data loss. The tree improvement arrange uses the connection accessibility, vitality utilization factor, in view of these qualities, the approaching hubs are powerfully include the system for dispensing with the rowdiness exercises. At long last, the built tree has been looked after simentanously, if the neighboring hub does not give any Reaction, considered to be the dead center, was expelled from the tree stage at that point. Therefore, the tree is preserved by comparing the remaining vitality and an estimate of the edge and relation of the device is conceived so that the data can be efficiently transferred from source to target. Then the steps of Fireflies Ant optimized reliable quality awareness energy efficient routing protocol (FAORQEER) based bio-medical data transmission process is discussed as follows.

Step 1: Gather the bio-medical sensor device based medical information.

Step 2: Analyze the incoming information and divided according to ordinary packet, middle packet and emergency packets.

Step 3: Calculate the path reliability value from the neighboring table information that is defined as follows,

$$
\boldsymbol{R}_{\text {path(i,Dis })=} \boldsymbol{R}_{\text {link }(i, j)} * \boldsymbol{R}_{\text {path(j,Dist })}
$$

Step 4: The computed node reliability is compared with neighboring node reliability value and choose the high reliability value related path.

Step 5: After that compute the link reliability value as follows,

$\boldsymbol{R}_{\text {link }(i, j)}=(1-\propto) \boldsymbol{R}_{\operatorname{link}(i, j)}+\propto \boldsymbol{X}_{\boldsymbol{i}}$

Step 6: Based on the probability value, the weighted average moving value is determined and selected path probability value is computed as follows,

$$
P_{i}^{k}(t)=\left\{\frac{\left|t_{i}(t)\right|^{\alpha}\left|n_{j}\right|^{\beta}}{\sum_{\mu}\left|t_{i}(t)\right|^{\alpha}\left|n_{j}\right|^{\beta}} \quad \text { if } i \epsilon^{k}\right.
$$

Step 7: According to the probability value, the distance between two path is measured as follows,

Step 8: Then the direction change of the path is estimated as follows.

$$
\text { attractiveness }=\left(\sum_{i=1}^{n}\left|x_{i}-y_{i}\right|^{p}\right)^{1 / p}
$$

$$
F_{i j}^{d}=G(t) \frac{M_{i}(t) * M_{j}(t)}{\left(D_{i j}(t)\right)^{n}+\varepsilon}
$$

Step 9: From the path direction, optimal path has been selected based on the fitness value that is computed as follows,

$$
F_{i}^{d}(t)=\sum_{j \epsilon k-\text { best } j \neq i} \operatorname{rand}_{j} F_{i j}^{d}(t)
$$

Step 10: After selecting the path, link reliability value, energy consumption of the node is computed.

Step 11: The computed node value, is organized in tree structure for making the transaction with level by level.

Step 12: At long last tree has been kept up if the hub does not answer for any demand; it thought to be dead hub and disposed of from the tree.

Else its neighboring quality is refreshed consistently and transmits the information.

Based on the above algorithm, the bio-medical data has been continuously transmitted that improves the reliability and quality of the node that reduces the network failure, node failure and link failure. Then the efficiency of the system is computed as follows.

\section{RESULTS AND DISCUSSIONS}

This section analyzes the efficiency of the Fireflies Ant optimized reliable quality awareness energy efficient routing protocol (FAORQEER) in wireless body sensor network The developed FAORQEER system implemented with the help of NS2 simulation tool which utilizes the real hospital scenario. Data have been obtained at a distance of 3 to 5 meters and are tested using the following parameter for simulation shown in Table 1 . The efficacy of this method will then be studied and compared to existing techniques such as LOCALMOR[ 15] and Energy Efficient Node Disjoined Multipath Routing 
Fireflies Ant Optimized Reliable Quality Awareness Energy Efficient Routing Protocol for Managing Quality of Medical Data in Wireless Body Networks

Protocol (EDEMRP) (Localized Multi-Objectives Routing Protocol) [18]

Table 1. Simulation Parameters

\begin{tabular}{|c|c|}
\hline Parameters & Values \\
\hline Simulation Area & $250 \mathrm{~m}^{2}$ \\
\hline Number of nodes & $\begin{array}{c}47 \text { node (40 sensor node, 3 sink node, 6 } \\
\text { relay node) }\end{array}$ \\
\hline MAC & IEEE 802.15.4 \\
\hline Packet size & 40 bytes \\
\hline $\begin{array}{c}\text { Transmission } \\
\text { rate }\end{array}$ & 250kbps \\
\hline $\begin{array}{c}\text { Frequencies } \\
\text { band }\end{array}$ & 420MHz,868MHz, 2.4GHz \\
\hline Channel mode & Log shadowing wireless model \\
\hline $\begin{array}{c}\text { Evaluation } \\
\text { Parameters }\end{array}$ & $\begin{array}{c}\text { Delay, Energy Utilization factor, packet } \\
\text { delivery ratio }\end{array}$ \\
\hline Simulation time & 400sec \\
\hline
\end{tabular}

Based on the above simulation setup, the efficiency of Fireflies Ant optimized reliable quality awareness energy efficient routing protocol (FAORQEER) is analyzed in terms of using end to end delay, energy utilization and packet delivery ratio which is discussed as follows.

\section{End to End Delay}

The metric used to measure the time to taken for transmitting data from source to sink node which measured as follows,

$$
\text { Delay }=Q D+P D+P G D
$$

\section{Where,}

$Q D$ is the queuing delay, $P D$ is processing de

\section{Energy Utilization Factor}

EUF is used to evaluate efficiently measured data transmission by the power consumption function.

$$
E U F=E U / T E \times 100
$$

\section{Packet Delivery Ratio}

Packet Delivery ratio is measure used to how effectively transmit the data to destination without making any delay.

$$
P D R=\frac{\text { No of packets transmitted successfully }}{\text { No of packets generated }} \times 100
$$

\section{Packet Loss Ratio (PLR)}

Packet loss ratio is metric that used to measure how the method transmit the information without losing any important information while making data transaction from source to destination.

According to the above metrics, the obtained end to end delay value is shown in table 2

\section{Table 2: End to End Delay}

\begin{tabular}{|l|l|l|l|}
\hline & \multicolumn{3}{l|}{ End to End Delay } \\
\hline $\begin{array}{l}\text { Number } \\
\text { of Nodes }\end{array}$ & LOCALMOR & EENDMRP & FAORQEER \\
\hline 10 & 57 & 52 & 32 \\
\hline 20 & 64 & 61 & 45 \\
\hline 40 & 79 & 65 & 53 \\
\hline 50 & 112 & 98 & 76 \\
\hline 70 & 147 & 121 & 85 \\
\hline 80 & 175 & 138 & 93 \\
\hline 100 & 210 & 169 & 104 \\
\hline 120 & 264 & 175 & 111 \\
\hline
\end{tabular}

The above table 2, clearly indicates that FAORQEER protocol ensures the minimum dealy $(74.87 \mathrm{~s}$-in average) when compared to the other methods such as LOCALMOR (138.5s) and EENDMRP (109.875s) while transmitting data from source to destination. Then the obtained delay value graphical representation is shown in figure 2 .

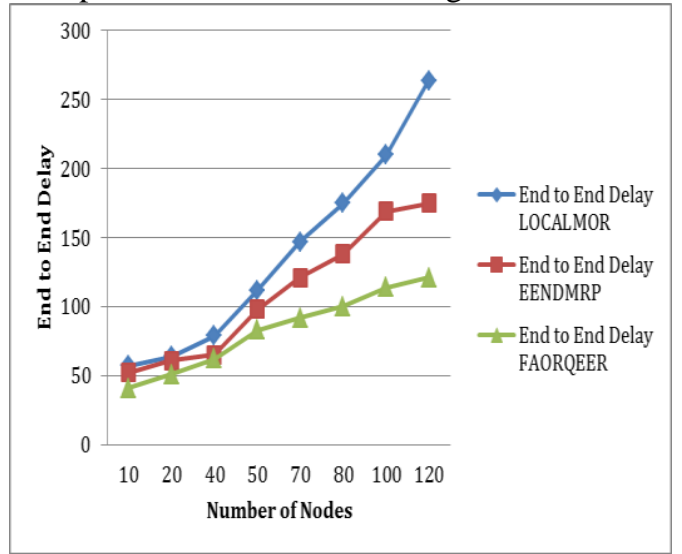

Figure 2: End to End Delay

Fig. 2 above indicates that in contrast with the traditional methods LOCALMOR and EENDMRP, the FAORQEER method requires a minimal delay. The minimum delay also raises the bio-sensor data transfer ratio for the transmission method of data in accordance with that priority and indicates the quality obtained in Table 3 .

Table 3: Packet Delivery Ratio

\begin{tabular}{|r|r|r|r|}
\hline $\begin{array}{l}\text { Number } \\
\text { of Nodes }\end{array}$ & LOCALMOR & EENDMRP & FAORQEER \\
\hline 10 & 83 & 85 & 95.36 \\
\hline 20 & 86.4 & 87.9 & 95.67 \\
\hline 40 & 88.2 & 91.3 & 97.13 \\
\hline is propa gation $\boldsymbol{d e z a} \boldsymbol{y}$. & 93.2 & 98.24 \\
\hline 70 & 88.8 & 92.5 & 98.8 \\
\hline 80 & 89.1 & 94.3 & 99.2 \\
\hline 100 & 85.6 & 91.2 & 99.44 \\
\hline 120 & 83.8 & 92.2 & 99.76 \\
\hline
\end{tabular}

The above table 3, clearly shows that FAORQEER attains high packet delivery ratio $(97.95 \%)$ compared to the other methods such as LOCALMOR $(83.8 \%)$ and EENDMRP $(92.5 \%)$ while transmitting bio-sensor data from source to destination. Then the obtained value is shown in figure 3 .

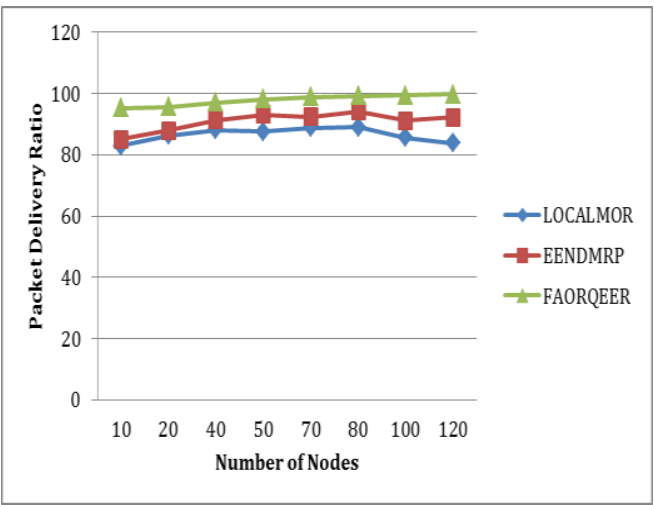

Figure 3: Packet Delivery Ratio

Based on the above figure 3, shows that effective transmission of bio-sensor data (ordinary, emergency and middle packets) with high packet delivery ratio. Even though the method consumes 
minimum energy when compared to other method which is shown in table 4.

Table 4: Energy Utilization Factor

\begin{tabular}{|r|r|r|r|}
\hline $\begin{array}{c}\text { Number } \\
\text { of Nodes }\end{array}$ & \multicolumn{1}{|c|}{ LOCALMOR } & EENDMRP & FAORQEER \\
\hline 10 & 3.5 & 4.1 & 1.98 \\
\hline 20 & 3.8 & 4.7 & 2.21 \\
\hline 40 & 4.1 & 5.2 & 2.32 \\
\hline 50 & 4.8 & 5.6 & 2.28 \\
\hline 70 & 5.3 & 6.4 & 3.21 \\
\hline 80 & 6.2 & 6.9 & 3.78 \\
\hline 100 & 7.2 & 8.3 & 4.23 \\
\hline 120 & 7.9 & 8.9 & 4.89 \\
\hline
\end{tabular}

The above table 4, clearly indicates that FAORQEER protocol ensures the high packet delivery by consuming lower energy (3.11J) when compared to the other methods such as LOCALMOR (5.5J) and EENDMRP (6.32J) while transmitting data from source to destination. Then the obtained value is shown in figure 4.

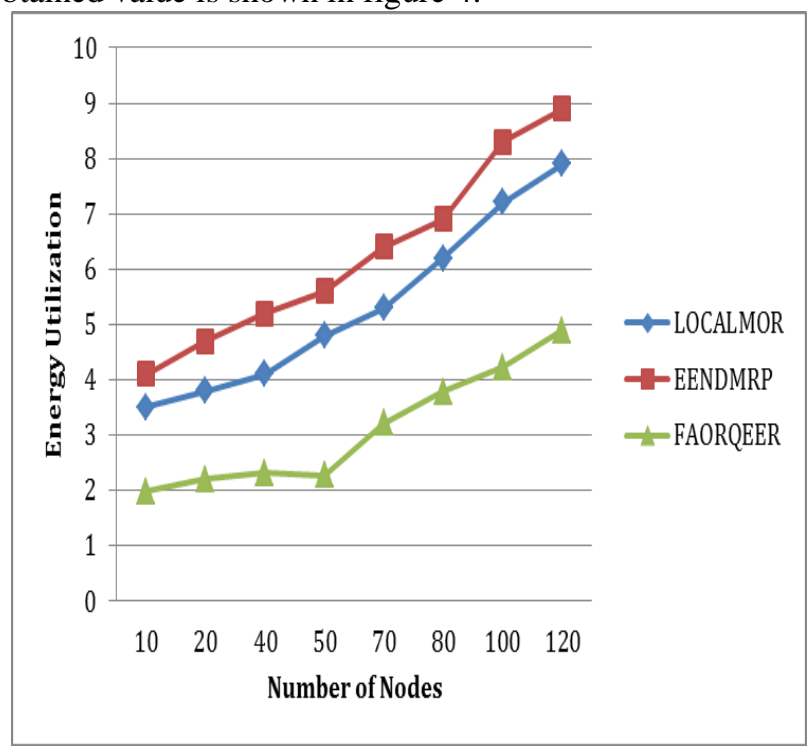

Figure 4: Energy Utilization Factor

Depending on the figure 4 , it clearly shows that FAORQEER system utilizes minimum energy that shows that network ability to withstand their lifetime while transmitting biomedical data from source to sink node. Although the FAORQEER method transmits the data by consuming minimum energy it should avoid packet loss value and the computed value is shown in table 5.

Table 5: Packet Loss Ratio

\begin{tabular}{|r|r|r|r|}
\hline $\begin{array}{l}\text { Number } \\
\text { of Nodes }\end{array}$ & \multicolumn{1}{|l|}{ LOCALMOR } & EENDMRP & FAORQEER \\
\hline 10 & 53 & 42.4 & 15.6 \\
\hline 20 & 56.4 & 47.29 & 15.27 \\
\hline 40 & 58.2 & 41.33 & 17.23 \\
\hline 50 & 57.5 & 43.62 & 18.2 \\
\hline 70 & 56.8 & 45.5 & 18.18 \\
\hline 80 & 59.1 & 44.3 & 19.12 \\
\hline 100 & 55.6 & 41.2 & 19.04 \\
\hline 120 & 53.8 & 42.62 & 19.26 \\
\hline
\end{tabular}

The above table 4, clearly shows that FAORQEER attains minimum packet loss ratio $(17.73 \%)$ compared to the other methods such as LOCALMOR (56.3\%) and EENDMRP $(43.53 \%)$ while transmitting bio-sensor data from source to destination. Then the obtained value is shown in figure 5

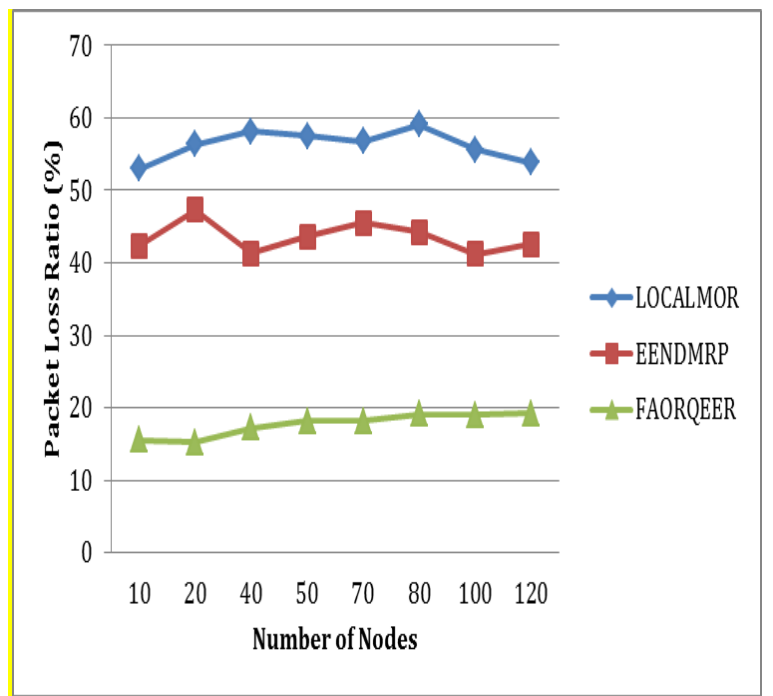

Figure 5: Packet Loss Ratio

Thus the Fireflies Ant optimized reliable quality awareness energy efficient routing protocol (FAORQEER) system efficiently transmits the patient information via the BWSN network with minimum delay, minimum energy and packet delivery ratio when compared to other routing methods.

\section{CONCLUSION}

This study examined Fireflies Ant for the broadcast of the bio-sensor data into wireless sensor networks and optimized reliable performance understanding of energy-efficient routing protocol (FAORQEER). At the time of data transmission, a reliability quality that helps to improve the sensitive overall process was examined according to the node and route.The reliability value is examined with the help of neighboring node information that minimize the data failures and other intermediate attacks. According to the reliability value, path has been selected and optimized path is chosen based on the fitness value. Based on the reliability value of node, link, energy consumption of the node is estimated to manage the network lifetime also reduce the data failure, network failure, node failure and link failure. Finally, the efficiency of the system is examined in terms of using simulation results in which FAORQEER attains minimum delay, low packet loss ratio that leads to enhance the overall packet delivery ratio also consumes minimum energy.

\section{REFERENCE}

1. Dong L., Wu J.K., Chen X. Real Time Physical Activity Monitoring by Data Fusion in Body Sensor Networks. Proceedings of the 10th International Conference on Information Fusion; Quebec, QC Canada. 9-12 July 2007.

2. Aida E., Massoud H., Pejman K. Using Relay Network to Increase Life Time in Wireless Body Area Sensor Networks. Proceedings of IEEE International Symposium on a World of Wireless, Mobile and Multimedia Networks \& Workshops; Kos, Greece. 15-19 June 2009; pp. 1-6.

3. Daisuke T., Xiao Y., Hu F., Chen J.M., Sun Y.X. Temperature-aware routing for telemedicine applications in embedded biomedical sensor networks. EURASIP J. Wirel. Commun. Netw. 2008;2008 doi $10.1155 / 2008 / 572636$.

4. Hachisuka K., Nakata A., Takeda T., Terauchi Y., Shiba K., Sasaki K., Hosaka H., Itao K. Development and Performance Analysis of an Intra-Body Communication Device. Proceedings of the 12th International Conference on Transducers, Solid-State Sensors, Actuators and 


\section{Fireflies Ant Optimized Reliable Quality Awareness Energy Efficient Routing Protocol for Managing Quality of Medical Data in Wireless Body Networks}

Microsystems; Boston, MA, USA. 8-12 June 2003;

5. Wegmueller M.S., Kuhn A., Froehlich J., Oberle M., Felber N., Kuster N., Fichter W. An attempt to model the human body as a communication channel. IEEE Trans. Bio-Med. Eng. 2007;54:18511857.

6. Xiaochen Lai, Quanli Liu, Xin Wei, Wei Wang, Guoqiao Zhou, and Guangyi Han, “A Survey of Body Sensor Networks”, Sensors (Basel). 2013 May; 13(5): 5406-5447.

7. Javed Iqbal Bangash, Abdul Hanan Abdullah, Mohammad Hossein Anisi, and Abdul Waheed Khan, "A Survey of Routing Protocols in Wireless Body Sensor Networks", Sensors (Basel). 2014 Jan; 14(1): $1322-1357$.

8. Zahoor Khan, Nauman Aslam, Shyamala Sivakumar, William Phillips, "Energy-aware Peering Routing Protocol for indoor hospital Body Area Network Communication", Procedia Computer Science,Volume 10, 2012.

9. Sharma, Vyas, Thakker, Mulvaney,Datta, "Wireless Body Area Network for health monitoring", International Conference on Biomedical Engineering and Informatics (BMEI), Volume:4 ,2011.

10. Shyamal Patel, Hyung Park, Paolo Bonato, Leighton Chan and Mary Rodgers, "A review of wearable sensors and systems with application in rehabilitation", Journal of NeuroEngineering and Rehabilitation20129:21

11. Wei Quan, Fu-Teo Zhao,Jian-Feng Guan, Chang-Qiao XU, and Zhang Hongke,"An Integrated Ling Quality Estimation-Based Routing For Wireless Sensor Networks,"Trans. on ELSEVIER, pp.28-33, 2011

12. DjamelDjenouri and Ilangko Balasingham,"Traffic- DifferentiationBased Modular QOS Localized Routing For Wireless Sensor Networks,"IEEE Transactions

Mobile Computing,vol.10,no.6,pp.797-809, 2011

13. Mellal, M. A., \& Williams, E. J. (2018). A survey on ant colony optimization, particle swarm optimization, and cuckoo algorithms. In Handbook of research on emergent applications of optimization algorithms (pp. 37-51). IGI Global. DOI:10.4018/978-1-5225-29903.ch002

14. Rashedi, E., Nezamabadi-Pour, H., \& Saryazdi, S. (2009). GSA: a gravitational search algorithm. Information sciences, 179(13): pp. 2232-2248

15. A. Rahim, N. Javaid, M. Aslam, Z. Rahman, U. Qasim, Z. A. Khan, "A Comprehensive Survey of MAC Protocols for Wireless Body Area Networks", available at. http://arxiv.org/pdf/1208.2351.pdf

16. Shiva Murthy G, R.J.DSouza, and Varaprasad G, "Reliability Analysis of Route Redundancy Model for Energy Efficient Node Disjoint Multipath Routing

Wireless sensor networks,"Trans. on ELSEVIER, in proc. ICMOC,pp.1487-1494, 2012.

17. DjamelDjenouri and Ilangko Balasingham,"Traffic- DifferentiationBased Modular QOS Localized Routing For Wireless Sensor Networks,'IEEE Transactions Mobile Computing,vol.10,no.6,pp.797-809, 2011

18. Shiva Murthy G, R.J.DSouza, and Varaprasad G, "Reliability Analysis of Route Redundancy Model for Energy Efficient Node Disjoint Multipath Routing

Wireless sensor networks,"Trans. on ELSEVIER, in proc. ICMOC,pp.1487-1494

2012 .

\section{AUTHORS PROFILE}

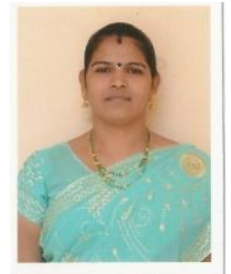

M.Santhalakshmi received her M.Sc from Periyar University, Salem, India in 2002, M.Phil from Periyar University, Salem, India in Jan 2007, MCA degree from Periyar University, Salem, India in March 2009. Currently she is an Assistant Professor in Department of Computer Science cum Vice Principal for Science Stream, Sri Kailash Women's College, Thalaivasal, Salem, Tamil Nadu, India. Her Research Interest include Networks, Wireless Networks, Mobile Computing, Wireless Sensor Networks.
Dr.P.Kavitha received her MCA from Bharathidasan University, Trichy, Tamil Nadu, India in 1999, M.Phil from Periyar University, Salem, Tamil Nadu, India in 2006, Ph.D from Periyar University, Salem, Tamil Nadu, India in 2016. Currently she is an Assistant Professor cum Head Of the Department in Department of Computer Science, Paavendhar college of Art's \& Science, Thalaivasal, Salem, Tamil Nadu, India. She has Authored 13 Papers in International journal And she Published 2 books in Multimedia Systems \& Advanced Java Programming in MSK Publications in 2009 \& 2010. Her research interest areas include Data mining, Mobile Computing, Hand off Signal Processing Networks. 